\title{
Comparison of Pulse Diagnosis in Oriental and Western Medicine
}

\author{
Kim Kyung-Won ${ }^{1}$ and Na Woo-Gwun ${ }^{2 *}$ \\ 'Department of Nursing, Daegu Haany University, Daegu - 706828, South Korea; \\ kyungwok@dhu.ac.kr \\ 2Department of Philosophy, Korea University, Seoul - 136701, South Korea; \\ laotao@chol.com
}

\begin{abstract}
There are marked differences between oriental medicine and western medicine on the pulse measurement and interpretation of abnormality. The purpose of this study was to examine and analyze methods of pulse palpation between oriental and western medicine. This study is an exploratory research to compare the meaning of pulse palpation, methods of pulse measurement, and interpretation on good health and bad health in oriental medicine and western medicine through pulse palpation. The pulse diagnosis in western medicine, depend on the pulse rate, rhythm, strength, and elasticity, anyone can do it by short-time training quite easily. The pulse diagnosis in oriental medicine is more comprehensive and more detailed method because it consists of the twenty-eight types of pulse and eighteen classes. It also depends on a doctor's subjective sensations and oral tradition. Therefore, nurses can utilize the advantages of oriental pulse palpation in assessing health status of patient; the quality of patient care will improve in the integrated care.
\end{abstract}

Keywords: Oriental Medicine, Pulse Diagnosis, Western Medicine

\section{Introduction}

Pulse palpation is an important indicator, it has been used to assess health status in people both oriental medicine and western medicine. Various health statuses of people have been assessed, distinguished abnormal from normal and analyzed by pulse palpation of oriental doctors. They found the parts where the disease began, analyzed the characteristics of the disease, judged the progress and the prognosis of the disease ${ }^{1,6}$.

In western medicine, pulse palpation is also a useful index to assess health status of client with body temperature, respiration, and blood pressure, it has been used for important documents to decide medical treatment and nursing intervention together with the usual health of client and the response on the physical and the psychological stress ${ }^{9,10}$. However, pulse palpation in oriental medicine is different from that in western medicine regardless of using wrist pulse diagnosis. It is reported that oriental medical doctors could predict where the disease began, characteristics of the disease, and the severity and the prognosis of disease by searching various forms of pulse in both wrists ${ }^{6}$.

In this paper, we will compare the methods of pulse palpation used by oriental medicine and western medicine daily in clinical settings to note the differences and similarities. Furthermore, we will consider that the pulse palpation of oriental medicine is not set in stone, but it has undergone various transformations in historical trends and it is also advancing.

This study will serve as momentum to fuse advantages of pulse palpation in oriental and western medicine and an approach in deep awareness various symptoms in patients by segmentation of types of pulse. It will be one of the processes that Korean nursing provide more quality of nursing care for patients.

\footnotetext{
* Author for correspondence
} 


\section{Methodology}

This study was conducted in two parts. First, exploratory research was done to compare the meaning of pulse diagnosis, methods of measurement, and characteristics and types of pulse related to health and disease in oriental and western medicine through pulse palpation. In presenting this argument, 'West' viewpoint was first described, according to the standard of western medicine, in case of the 'Oriental', after marking the oriental, we described it following in the west. If there were no differences, we did not describe it. Second, we are considered that method of pulse palpation which was used by oriental doctors in the traditional ages.

\section{Findings}

\subsection{Meaning of Pulse Palpation}

The pulse is the bounding of arterial blood flow that palpable at different points on the body. The pulse means the stroke volume, ejection and distension of the walls of the aorta, which creates a pulse wave as it travels rapidly toward the distal ends of arteries. As the pulse wave reaches a superficial peripheral artery and travels over an underlying bone or muscle. The pulse can be palpated by applying gentle pressure over a pulse point ${ }^{9,10}$.

The heart is a muscular organ for pumping blood. The left ventricle of the heart contracts with every beat, forcing blood from the heart into the systemic arteries. The amount of blood pumped from the heart with each heartbeat is called the stroke volume. The force of the blood against the walls of the arteries generates a wave of pressure that is felt at various points in the body as a pulse ${ }^{9,10}$.

Unlike western medicine, oriental medicine was not limited to the pulse is a movement of the artery by pumping of the heart. Oriental doctors perceived that the body's vital currents appeared by wrist pulse palpation, the pulse is taken as a major index of the body condition of people. The pulse is always taken in three positions and on both sides, and by applying three different pressures superficial, medium, and deep each matching the quality of qi in two of the twelve key organs (Figure 1) ) $^{3,7}$.

left 1: heart/small intestine

left 2: liver/gall bladder

left 3: kidney/bladder right 1: lungs/large intestine

right 2: spleen/stomach

right 3: pericardium/triple heater.

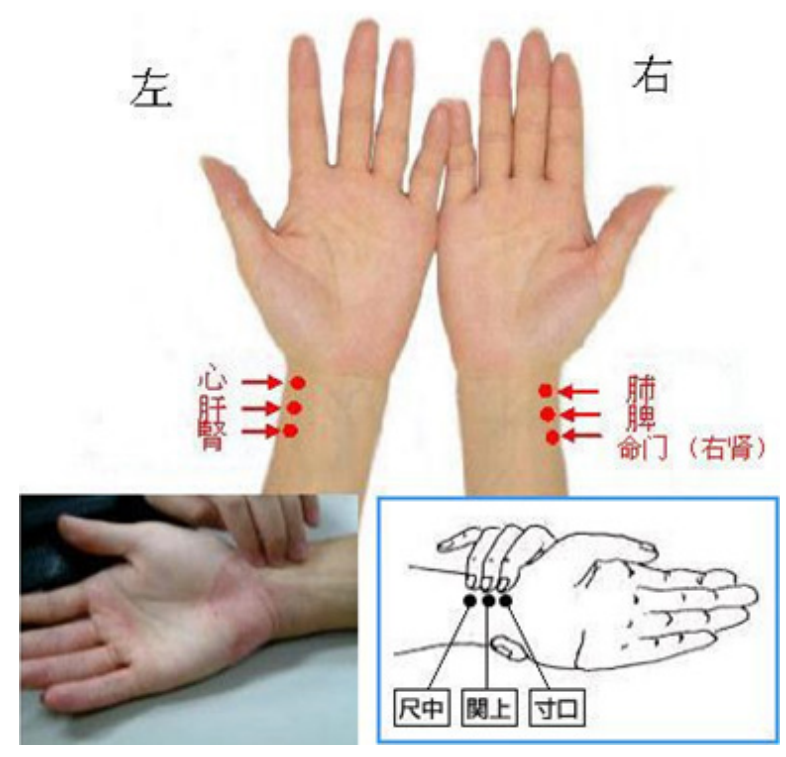

Figure 1. Organs and position of pulse palpation.

\subsection{Location and Methods of Measurement in Pulse Palpation}

The peripheral pulse site most used is the radical pulse. Radical pulse is palpated by placing the pads of the first two or three fingers on the anterior wrist along the radius bone. If the pulse is regular, the nurse counts the beats for 30 seconds and multiplies by 2 to obtained total bpm. If the pulse is irregular, the nurse counts the beats for full minute. Four factors are considered when assessing the pulse: rate, rhythm, force, and elasticity ${ }^{9,10}$.

In oriental medicine, measuring pulse also conducted in a stable condition with the height of your arm match the height of your heart.

The pulse most commonly used is the radial pulse too. The radial pulse is subdivided into three divisions from the wrist crease upward along the arm and parted into three depths. This creates eighteen positions that are crossreferenced to twenty-eight possible quality assessments. The twenty-eight types of pulses, then, consist of eighteen classes distinguished according to depth (2), speed (2), width (2), strength (2), shape (4), length (2), rhythm (3), and balance (1); plus ten types, i.e., flooding, minute, frail, soggy, leathery, hidden, confined, spinning, hollow, and scattered. The most desirable pulse is moderate, regular, medium, strong, and even ${ }^{7,8}$. 
Table 1. Characteristics of Pulse

\begin{tabular}{lll}
\hline Characteristics & \multicolumn{1}{c}{ Feeling } & \multicolumn{1}{c}{ Indication } \\
\hline superficial & easily palpable but weak & beginning disease \\
deep & felt only with pressure & internal syndromes \\
slow & less than four beats & cold syndromes \\
rapid & more than five beats & heat syndromes \\
taut & forceful and tight & yang hyperactivity \\
wry & rolling or flowing & excessive phlegm \\
thready & rather fine pulse & deficient qi/ blood \\
short & uneven, missing beats & qi / blood-stagnation \\
knotted & slow, irregular misses & endogenous cold \\
intermittent & slow, regular misses & impairment of qi \\
\hline
\end{tabular}

\subsection{Pulse Characteristics and Pulse Types}

In western medicine, characteristics that influence pulse include rate, rhythm, strength, and elasticity and these same features apply to oriental medicine (Table 1 and 2).

\subsubsection{Pulse Rate:}

A normal pulse rate for adults in a stable state is between 60 and 100beat per minutes. The pulse rate varies with age and gender. Pulse rate of infants and children is higher than that of adults and old people. The average pulse rate of the adult female is slightly higher than that of the adult male. In an adult, the heart rate less than 60 beats per minute is bradycardia, the heart rate excess of 100 beat per minute is tachycardia. In particular, pulse rate increases with exercises, response to stress, fear, and anxiety ${ }^{9,10}$.

In oriental medicine, a normal pulse rate for adults is also between 72 and 80 beat per minute. Pulse rate of less 4 times during one respiration is slow pulse; pulse rate of over $5 \sim 6$ times during one respiration is fast pulse. The younger, the pulse goes quickly, neonates are 7 8 times and infants are $6 \sim 7$ times in a breathing. In case of females, the pulse is slightly fast that of males. The pulse with fast or slow characteristics are rapid pulse(數脈) and slow pulse (逰脈 $)^{7,12}$.

\subsubsection{Pulse Rhythm:}

The rhythm is constant on a regular basis. However, sinus arrhythmia can appear in childhood and early adulthood. It is subject to changes according to cycles of respiration. Pulse rhythm speed up at the peak of inhalation, it speed down during exhalation, and then it returns to regular rhythm. Because the volume of pulse rate in the left ventricle of heart was reduced by the exhalation. In a moment, the pulse rate increase to compensate $i^{9,10}$. In oriental medicine, the pulse rhythm is better to equal. Abnormal characteristics of pulse rhythm also include intermittent pulse(代脈), bound pulse (結脈), and skipping pulse (促脈) $)^{3,8}$.

\subsubsection{Pulse Strength:}

It is the volume of force exerted by the ejected blood against the arterial wall with one contraction. It is described as pulse of weak means that decrease of the cardiac output (hypovolemic shock) and pulse of strong means that increase of the cardiac output, mainly it appears when we feel uneasy, do exercise or got in unhealthy status. Pulse strength is evaluated by 4-point scales;

$3+$ strong (bounding),

$2+$ normal (full, easily palpable),

$1+$ weak (thready and unusually rapid), and 0 non absent ${ }^{9,10}$.

In oriental medicine, pulse in adulthood is a full of energy (strong) and pulse in old age is weak; the pulse of the female is weaker than that of male. Having eaten and drunken too much, the pulse is fast and strong. When we are starving, the pulse is relatively weak. Abnormal characteristics of pulse strength divided into vacuous pulse $「$ 虛脈 $「$ and replete pulse $(\text { 實脈 })^{8}$.

While having a similar shape, the pulse classify the following types: surging pulse (洪脈), fine pulse (細脈), relaxed pulse (緩脈), faint pulse (微脈), weak pulse (弱 脈), stirred pulse (動脈) $)^{8}$.

\subsubsection{Elasticity of Pulse:}

It described that normal pulse is light and tight. In oriental medicine, the types of pulse elasticity divided into stringlike pulse(弦脈)and tight pulse(緊脈).

On the other hand, in contrast to the western medicine, oriental medicine, there are classification methods depending on the depth of pulse. They are floating pulse( 浮脈) sunken pulse(沈脈) dissipated pulse(散脈) firm pulse(牢脈) hidden pulse (伏脈) ${ }^{7,8}$.

As mentioned above, in a respect of classification, the pulse palpation in oriental medicine is more varied than that of western medicine.

\subsection{Transition of Pulse Palpation in Oriental Medicine}

There are four principles for the pulse palpation in Huangdi Neijing(黄帝內經). First, practitioner should 
feel the pulse of patient after a stable status. Second, practitioner should take patient's pulse in a comfortable position without a prejudice. Third, the location of pulse palpation has been changed from the whole body to the upper and lower body. Last, one finger mainly used to distinguish the types of pulse. Since chon, gwan and cheok (寸關尺) were classified by Nanjijn(難經), practitioners will use to three fingers. After all, the number of sites of pulse palpation decreased and practitioner learned how to use three fingers ${ }^{4}$.

On the site of pules palpation, there are four methods of pulse palpation in Huangdi Neijing(黃帝內 經), namely: Dong Macjin(動脈法), Three Bu Nine Hu Macjin(三部九候脈法), In Young Gi Gu Macjin(人迎氣 口脈法). Each method developed in its own way, but they are closely related and became the basis of today's pulse palpation. Detailed process is a starting point of arteries in the whole body, Dong Macjin(動脈法). The body is subdivided into three divisions from the wrist crease upward along the arm and parted into three depths since then.

As a result, Three Bu Nine Hu Macjin(三部九候脈法) has been developed and the radial artery was confirmed as the main site of pulse palpation and three fingers used to palpate pulse $^{2}$. This creates eighteen positions that are cross-referenced to twenty-eight possible quality assessments, eventually resulting in a diagnostic pattern? And it consist of the twenty-eight types of pulses eighteen classes distinguished according to depth, speed, width, strength, shape, length, rhythm, and balance with ten types. However, due to its complexity, it takes decades to become skilled at pulse diagnosis. It also depends on a doctor's subjective sensations and oral tradition ${ }^{5}$.

Because the unexpected problem arose, it took practitioner a long time to learn this method of pulse palpation, moreover, it was difficult to distinguish types

Table 2. Types of Pulse $e^{11}$

\begin{tabular}{|c|c|}
\hline Types & Definition (WHO International standard) \\
\hline Rapid Pulse(數脈) & a pulse with more than five or six beats to one cycle of the doctor's respiration, the same as tachycardia \\
\hline Slow Pulse (遲脈) & a pulse with less than four beats to one cycle of doctor's respiration, the same as bradycardia \\
\hline Intermittent Pulse(代脈) & a moderate weak pulse, pausing at regular intervals \\
\hline Bound Pulse(結脈) & a moderate weak pulse, pausing at irregular intervals \\
\hline Skipping Pulse(促脈) & a rapid pulse with irregular intermittence \\
\hline Slippery Pulse(滑脈) & $\begin{array}{l}\text { a pulse coming and going smoothly beads rolling on a plate -phlegm-retention syndrome, retention of } \\
\text { food resulting, fever due to excess, pregnancy }\end{array}$ \\
\hline Rough Pulse(涉脈) & a pulse coming and going unsmoothly with small, fine, slow joggling tempo like scraping bamboo with a knife \\
\hline Vacuous Pulse(虛脈) & a general term for a feeble and void pulse \\
\hline Replete Pulse(實脈) & a general term for a pulse felt forceful at all three parts, chon gwan and cheok (寸關尺), also forceful pulse \\
\hline Surging Pulse(洪脈) & a pulse beating like dashing waves with powerful rising and gradual decline, also called flooding pulse \\
\hline Fine Pulse(細脈) & $\begin{array}{l}\text { a pulse as thin as a silk thread, straight and soft, feeble yet always perceptible upon hard pressure, also } \\
\text { called thin/thread pulse }\end{array}$ \\
\hline Relaxed Pulse(緩脈) & a pulse with decreased tension \\
\hline Faint Pulse(微脈) & a feeble and soft pulse, hardly perceptible \\
\hline Weak Pulse(弱脈) & a pulse that is deep, soft, thin and forceless \\
\hline Stirred Pulse(動脈) & a quick, jerky pulse, like a bouncing pea, slippery, rapid and forceful \\
\hline String-like Pulse(弦脈) & a straight, long and taut pulse, like a musical string to the touch : disease of biliary system, pain, malraria \\
\hline Tight Pulse(緊脈) & a pulse feeling like a tightly stretched cord \\
\hline Floating Pulse(浮脈) & a superficially located pulse which can be felt by light touch and grows faint on hard pressure \\
\hline Sunken Pulse(沈脈) & a deeply located pulse which can only be felt when pressing hard, also called deep pulse \\
\hline Dissipated Pulse(散脈) & a pulse that feels diffusing and feeble upon a light touch and faint upon hard pressure \\
\hline Firm Pulse(牢脈) & a broad, forceful and taut pulse, deeply seated and felt only by hard pressure \\
\hline Hidden Pulse(伏脈) & a pulse which can only be felt upon pressing to the bone, located deeper than sunken pulse or even totally hidden \\
\hline
\end{tabular}


of pulse exactly. Present, eight types of pulse are utilize in clinical setting for pulse palpation.

Because professional also can't clearly distinguish various types of pulse.

\section{Conclusion}

Doctors in western and oriental medicine stick to the radical pulse palpation. Although they palpated the pulse by the same radical artery sites, the evaluation of diagnosis is different. It is a long tradition of oriental medicine and western medicine that they took advantage of patient's radical pulse palpation and diagnosed the disease.

When assessing the pulse in western medicine, pulse rate, pulse rhythm, strength, and elasticity of the pulse are considered, anyone can do it by short -time training quite easily. If abnormalities of pulse are found, they can use an electrocardiogram or an echocardiogram for an indepth assessment. Even if the pulse diagnosis in oriental medicine considered more comprehensive and more detailed method of health assessment than that in western medicine, it has difficult to utilized generally.

Recently, oriental medical doctors eager to measure forms of pulse and compile a database by using the medical engineering for the algorism of traditional pulse palpation ${ }^{6}$.

Nurses palpate pulse of many patients almost every day at the nearest them. Skilled nurses with many experiences of pulse palpation have more favorable terms to blend a detailed diagnostic method in oriental medicine by reinforcing a simple framework for understanding of pulse palpation in western medicine. If skilled nurses can apply the method of pulse palpation of oriental medical doctors to patient's care, they can detect high risk conditions of patient as soon as possible. It will increase the quality of life in patient with a speedy care. Therefore, It will be a trigger for search the characteristics of Korean nursing ${ }^{3}$.

\section{References}

1. Bilyion K, Hammer L, Zaslawski C. Contemporary Chinese pulse diagnosis: A modern interpretation of as ancient and traditional method. Journal of Acupuncture Meridian Study. 2013; 6(4):227-33.

2. Hur KB, Yoon JH, Kim KS. A study on three bu nine hu pulse diagnosis. The Journal of Korean Acupuncture and Moxibustion Society. 2002; 19(2):1-13, 2002.

3. Ha HY, Kim JA, Kim AJ. Oriental Nursing. Hanmibooks: Korea; 2010.

4. Jang YW. A study on the method of Macjin in the Whang Di Nei Qing. The Journal of Korean Medical Classics. 1998; 11(2):146-68.

5. Kaptchuck TJ. The web that has no weaver: Understanding Chinese medicine. 2nd ed. New York: Congdon \& Weed; 2000.

6. Kim HY, Kim JY, Kim YJ, Park YB. Development of pulse diagnostic device in Korea. Integrative Medicine Research. 2013; 2(1):7-17.

7. Kohn L. Health and longlife: The Chinese way. Three pines press: USA; 2005.

8. Park CK, Jun YJ, Kim NI, et al. Korean-English dictionary of oriental medicine. Paju: Jimoondang; 2004.

9. Sneed N, Hollerbach A. Accuracy of heart rate assessment in atrial fibrillation. Heart and Lung. 1992; 21(5):427-33.

10. Song KA, Park HS, Hong YA. Fundamentals of nursing. Korea: Soomoonsa; 2009.

11. WHO. International standard terminologies on traditional medicine in western pacific region: WHO; 2007.

12. You SB. A study on The Characteristic of Traditional Pediatric Pulse Diagnosis. The Journal of Korean Medical Classics. 2014; 27(1):111-22. 